Volume 2

Issue 4 -- Integrative Medicine

Article 30

$11-20-2015$

\title{
Disease-Management in Family Medicine Clinics Through the Addition of a Health Coach: A Pilot Study
}

Crystal Y. Cichon

Jessica J.F. Kram

Tiffany A. Mullen

Pamela Voelkers

Kristin J. Magliocco

Kiley A. Bernhard

Dennis J. Baumgardner

Follow this and additional works at: https://aah.org/jpcrr

Part of the Cardiovascular Diseases Commons, Endocrinology, Diabetes, and Metabolism Commons, and the Family Medicine Commons

\section{Recommended Citation}

Cichon CY, Kram JJ, Mullen TA, Voelkers P, Magliocco KJ, Bernhard KA, Baumgardner DJ. DiseaseManagement in Family Medicine Clinics Through the Addition of a Health Coach: A Pilot Study. $J$ Patient Cent Res Rev 2015;2:212-213. http://dx.doi.org/10.17294/2330-0698.1240

Published quarterly by Midwest-based health system Advocate Aurora Health and indexed in PubMed Central, the Journal of Patient-Centered Research and Reviews (JPCRR) is an open access, peer-reviewed medical journal focused on disseminating scholarly works devoted to improving patient-centered care practices, health outcomes, and the patient experience. 
nonselectively, inhibits fungal growth in soil samples at moderately high levels. It does not appear likely that this Scedosporium apiospermum strain employs $\beta$-Th resistance for selective advantage in cedar mulched landscaping.

\section{Mailed At-Home FIT Intervention to Increase Colorectal Screenings at Sixteenth Street Community Health Centers}

\author{
Alexander V. Herrera, Brian Hilgeman, Michelle Buelow, \\ Melissa A. Lemke
}

TRIUMPH Program, University of Wisconsin School of Medicine and Public Health; Internal Medicine and Family Medicine, Sixteenth Street Community Health Center

Background: Mailed at-home FIT intervention kits to increase colorectal cancer screenings at Sixteenth Street Community Health Centers (SSCHC).

Purpose: It is our goal to increase the current SSCHC colorectal cancer baseline screening rate of $23 \%$ to $50 \%$ within three years of full at-home FIT kit implementation.

Methods: Colon cancer is the second and third most common cause of cancer death in the United States in Hispanic men and women, respectively. Colonoscopy is the most common method of colon cancer screening, even among low-income patients. However, it has been shown in community health centers that mailed FIT kits are a more effective outreach method (40.7\% completion) than colonoscopy outreach $(24.6 \%)$ or usual care $(12.1 \%)$. We hope to increase colorectal cancer screening in eligible patients at the SSCHC through mailed at-home FIT kits that have FIT materials, instructions and educational materials based on the Health Belief Model. Results: A trial intervention will assess the potential for annual implementation with hopes of full implementation to all of SSCHC eligible patients in the future.

Conclusion: Application of culturally relevant interventions can be a practical and inexpensive method of increasing colorectal screening rates in community health centers with predominantly Hispanic populations.

\section{Maternal Intuition of Fetal Gender}

Michael P. McFadzen, David P. Dielentheis, Ronda Kasten

\section{Department of Obstetrics, Aurora Sheboygan Clinic}

Background: Many pregnant mothers feel they have a perception or intuition as to the gender of their unborn baby. There is very little published scientific literature regarding this topic. The study's goal is to determine accuracy of mothers' perceptions as to gender of their unborn babies. Many scientists believe a pregnant woman could not determine her baby's gender by intuition, with a $50 \%$ probability of correctly determining the gender. This study should be considered fun science.

Purpose: To objectively measure a pregnant mother's perception as to the gender of her unborn baby and compare to sonographically proved gender. The study also will measure the percentage of pregnant patients who have this intuition.

Methods: All patients will be presenting for their secondtrimester screening ultrasound in the Obstetrics Department of Aurora Sheboygan Clinic and must be 17-23 weeks pregnant. A medical sonographer will describe the ultrasound exam and obtain appropriate consent and medical history. The patient will be asked if they have perception as to the fetal gender; their answer will be logged. Patients with knowledge of fetal gender will be excluded from this study. Results: Thus far, 128 patients have qualified for the study (with an expected cohort of 400). Approximately one-third of our patient population has "intuition" or "perception" on the gender of their baby. Of these, $47 \%$ correctly indicated fetal gender, 53\% did not. Within this study, we've started categorizing patients who have a strong intuition of fetal gender. This cohort has correctly indicated gender with $90 \%$ accuracy; however, there are not enough participants for clinical relevancy at this point in the study.

Conclusion: Preliminary data indicates mom perception of fetal gender is $47 \%$ accurate.

\section{Disease-Management in Family Medicine Clinics Through the Addition of a Health Coach: A Pilot Study}

Crystal Y. Cichon, Jessica J.F. Kram, Tiffany A. Mullen, Pamela Voelkers, Kristin J. Magliocco, Kiley A. Bernhard, Dennis J. Baumgardner

Department of Family Medicine, Aurora Health Care; Center for Urban Population Health; Aurora Advanced Health Care; University of Wisconsin School of Medicine and Public Health; Aurora UW Medical Group

Background: In the United States, more than $80 \%$ of health care spending is focused on the management of chronic illnesses such as hypertension, diabetes and hyperlipidemia. Controlling these chronic diseases can lead to better health outcomes and decrease the number of preventable deaths. Patient self-management has shown to improve clinical outcomes. In a primary care setting, a multidisciplinary approach can more effectively educate patients on improving their health.

Purpose: To assess the impact of a health coach in a primary care setting as it relates to clinical outcomes.

Methods: Patients from two Aurora family medicine clinics were referred to a health coach by primary care providers. A total of 40 patients participated and paid out of pocket for the health coaching sessions (intervention). Patients had at least one scheduled session with the health coach that covered topics such as healthy eating, weight loss and exercise. Patient data, including glycohemoglobin, lipid panels and blood pressures, were reviewed pre- and postintervention. 
Data were obtained 1 year before the intervention date and at least 3 months after. Paired t-tests were used for comparisons. Results: The study population was predominantly Caucasian $(90 \%)$ and female $(90 \%)$ with a mean age of 54 years (range 25-79). The mean patient body mass index $\left(\mathrm{kg} / \mathrm{m}^{2}\right)$ was 37 and ranged from 28 to 63 . When comparing pre- and postintervention clinical data, several improvements in laboratory values were noted. Low-density-lipoprotein cholesterol levels decreased from an initial mean of 114 preintervention to 105 postintervention, mean high-densitylipoprotein cholesterol levels increased from 47 to 58, and mean glycohemoglobin levels decreased from 6.5 to 6.1 . All improvements in clinical data were not statistically significant, but were clinically relevant.

Conclusion: Patients showed mild improvements in multiple lab values after their first meeting with a health coach. This pilot study was limited by the small number of patients who chose to have a health coaching session. A limiting factor for patient use of a health coach may be secondary to the cost of each clinic visit and follow-up lab work. Cost may have contributed to our demographic mix. To further assess the impact and benefit of a health coach in a primary care setting, a larger, more diverse patient population is needed.

\section{Real-World Relevance of Manual Electrocardiography QT Interval Measurement}

Satish Velagapudi, Zahra Nur Khaled, Bilal Omery, Firas Zahwe, Michael Anigbogu, Sarah Zukkoor, Indrajit Choudhuri

Department of Cardiology, Aurora Health Care; Department of Pharmacy, Aurora St. Luke's Medical Center; Aurora Cardiovascular Services, Aurora Health Care

Background: Electrocardiography (ECG) QT interval (QTI) prolongation independently predicts sudden death. Hospitalized patients are commonly exposed to multiple QT-prolonging drugs, and manual measurement of ECG QTI based on identifying the intersection of isoelectricity with the tangent to the terminal phase T-wave slope (QTTTT) is advocated due to inaccuracies in automated detection algorithms that may imprecisely identify QT duration.

Purpose: We evaluated the performance ofQTTTT compared to a standard automated (12SL, GE Healthcare) method (QT-12SL).

Methods: Consecutively obtained ECGs of 250 hospitalized patients were reviewed. The QTI in leads II, aVR, V5 and V6 determined by QT-12SL and QTTTT were compared. ECGs in which QT-12SL and QTTTT differed by $>10 \mathrm{~ms}$ were further characterized.

Results: The T-wave end was not reliably identified in 6 ECGs (2.4\%). Of the remaining 244 ECGs (976 leads), QTTTT differed from QT-12SL by $<10 \mathrm{~ms}$ in 52 ECGs $(21.3 \%)$. QT-12SL differed from QTTTT by $>10 \mathrm{~ms}$ in lead II in 140 leads (14.3\%), V5 in 149 leads (15.3\%), V6 in 152 leads $(15.6 \%)$ and aVR in 143 leads (14.7\%). ECGs with mutually exclusive lead combinations in which QTTTT differed from QT-12SL by > $10 \mathrm{~ms}$ were: 1) II, aVR, V5, V6 (39.8\%); 2) V5, V6 (7.8\%); 3) II, aVR (4.9\%); and 4) II, V5, V6 (3.7\%). The expected overestimation of QTI by QT-12SL compared to QTTTT exceeded $10 \mathrm{~ms}$ in 105 leads (10.8\%), related to T-waves with "normal" appearance, or biphasic (negative-to-positive) morphology; U-wave; and TP segment voltage exceeding PR segment voltage. Compared to QTTTT, QT-12SL underestimated QTI in 479 leads $(49.1 \%)$, in association with biphasic T-waves (positiveto-negative); atrial arrhythmias; downsloping baseline near the T-wave end resulting in TP segment voltage less than PR segment voltage; and slow return of T-wave terminus to baseline.

Conclusion: Multiple clinical and electrical phenomena impacted automated QTI determination. QT-12SL and QTTTT were comparable across all analyzed leads in only 1/5 of ECGs. Compared to QTTTT, QT-12SL QTI determinations were discordant in $3 / 5$ of all leads, and underestimated QTI nearly half the time. Perhaps most important, for a given ECG, manual review of any of the analyzed leads identified these differences $2 / 3$ of the time.

\section{Aligning Asthma Education Across the Continuum of Physician Education: Impact on Clinical Metrics}

Lisa Sullivan Vedder, Deborah Simpson, Jacob L. Bidwell, John R. Brill, Theresa Frederick

Departments of Family Medicine and Academic Affairs, Aurora UW Medical Group; Office of Continuing and Professional Development, Aurora Health Care

Background: All trainees entering family medicine residency training programs after June 1, 2012, must complete the same American Board of Family Medicine (ABFM) Maintenance of Certification (MOC) requirements as practicing physicians. These shared requirements provide an opportunity to align physician education initiatives across the continuum focused around a clinical care topic to improve health care system metrics.

Purpose: To assess the initial effectiveness of an ABFM Asthma Part IV approved MOC module, aligned to meet residency and medical student program accreditation requirements, on health care system metrics.

Methods: An ABFM Asthma Part IV MOC module was implemented for family medicine physicians and residents in April 2014 with open, rolling enrollment for all providers. The module focused on a $20 \%$ asthma control test (ACT) improvement as ACT is a potential driver for appropriate use of asthma controller medications (ACM) in persistent asthma and completion of the asthma action plan (AAP). Students rotating on a required primary care clerkship received a 1-hour orientation to quality improvement principles and their role in assuring that an ACT had been completed on their patients. Care quality measures at baseline (January 2014) and 12 months later (December 2014) were compared: ACT use, AAP completion, and percentage of patients on 